CASE REPORT

\title{
Resolution of chronic severe refractory thrombocytopenia after treatment of hypothyroidism
}

\author{
K M Bowles, G E Turner, J Z Wimperis
}

J Clin Pathol 2004;57:995-996. doi: 10.1136/icp.2004.016543

The case of a 52 year old woman with chronic severe refractory thrombocytopenia is presented. Over a three year period, her platelet count was persistently less than $20 \times 10^{9} /$ litre (normal range, 150-400). She required repeated hospital admission for management of bleeding and received multiple blood transfusions. She was given repeated courses of steroids, immunosuppression, immunoglobulin, and splenectomy, without success, in an attempt to stop the chronic blood loss. Eventually, she was found to be profoundly hypothyroid. On correction of her thyroid deficiency the platelet count returned to the normal range and all bleeding stopped. The platelet count remains in the normal range three years later.

diopathic thrombocytopenia purpura (ITP) is an autoimmune disorder characterised by persistent thrombocytopenia (platelet count, $<150 \times 10^{9}$ /litre) as a result of autoantibody binding to platelet antigens and causing their premature destruction by the reticuloendothelial system, in particular the spleen. ${ }^{1}$ We present the case of a patient with chronic, life threatening ITP and hypothyroidism, whose thrombocytopenia resolved completely after thyroid hormone replacement treatment.

\section{CASE REPORT}

A 52 year old woman was referred to the haematology clinic for investigation of thrombocytopenia. Her platelet count had failed to recover two months after receiving platinum based chemotherapy for relapsed ovarian carcinoma and she was suffering from episodes of rectal bleeding, epistaxis, and widespread bruising. She was on no regular medication and hepatitis and human immunodeficiency virus serology were negative. Her full blood count was unremarkable other than a platelet count of $17 \times 10^{9}$ /itre. Blood film confirmed thrombocytopenia and no red cell fragmentation. Serum creatinine, calcium, bilirubin, albumin, prothrombin time, and activated partial thromboplastin time were all within the normal range. A bone marrow biopsy showed a normocellular marrow with megakaryocytes that were normal in morphology and number. There was no evidence of infiltration or bone marrow disease, and by exclusion a diagnosis of ITP was made. Computerised tomography scans and serial $\mathrm{Ca}^{125}$ measurements confirmed clinical remission of her ovarian carcinoma.

Over the following year the patient was treated with courses of steroids, azathioprine, intravenous immunoglobulin, and Tranexamic acid without clinical or haematological improvement. She continued to have melaena, bruising, and epistaxis and required monthly admissions for blood transfusion. Gastrointestinal endoscopies revealed diffuse blood loss and failed to identify an anatomical cause for the bleeding. Helicobacter pylori was not diagnosed and at no point was she given $H$ pylori eradication treatment. The bleeding was attributed to the persistently low platelet count (less than $20 \times 10^{9}$ /litre).

She was then admitted with a massive gastrointestinal bleed that failed to resolve with conservative treatment and an emergency colectomy was performed. Surgery was covered with multiple platelet transfusions, but no incremental rise was seen in her platelet count. During the procedure, the spleen was removed but this failed to improve the platelet count. Over the next 12 months further courses of steroids and immunosuppression were tried without success; transfusion requirements increased to approximately three units of red blood cells each month and the patient became increasingly fatigued and depressed. She lost $5 \mathrm{~kg}$ in weight and reported thinning of her hair. Thyroid function was assessed and she was found to be profoundly hypothyroid (free thyroxine, $<1 \mathrm{pmol} /$ litre (normal range, 9-20), thyroid stimulating hormone (TSH), > $65 \mathrm{miu} /$ litre (normal range, 0.4-4.0)). She was started on thyroxine, after which there was an immediate clinical improvement in the severity and frequency of the bleeding. After three months of treatment, her TSH was suppressed to within the normal range and her platelet count had risen to $36 \times 10^{9}$ /itre. After six months of thyroxine, her TSH was suppressed to below $1 \mathrm{miu} / \mathrm{litre}$ and her platelet count had risen to $520 \times 10^{9}$ /litre, with complete resolution of all bleeding symptoms. Over the subsequent three years, the patient has suffered no further problems with bleeding and the platelet count has remained within the normal range.

\section{DISCUSSION}

The diagnosis of ITP is based principally on the clinical history, physical examination, full blood count, and examination of the peripheral blood smear, which should exclude other causes of thrombocytopenia. ${ }^{12}$ In adults, spontaneous remissions are rare, with ITP tending to run a chronic protracted course despite treatment. Bone marrow biopsy, if performed, should be normal. Antiplatelet antibodies are not useful diagnostically.

\section{"Autoantibodies to platelet antigens have been shown to coexist with autoimmune thyroid disease"}

There is no single diagnostic test for ITP, but we believe that the clinical findings and laboratory results in our patient were entirely consistent with a diagnosis of ITP. Over a three year period, our patient failed to respond to repeated courses of several medical treatments and splenectomy. However, with correction of her thyroid deficiency and adequate suppression of her TSH the platelet count returned to normal and the bleeding symptoms resolved completely. After three

Abbreviations: ITP, idiopathic thrombocytopenia purpura; TSH, thyroid stimulating hormone 


\section{Take home messages}

- We describe the case of a 52 year old woman with chronic severe refractory thrombocytopenia

- She required repeated hospital admission for management of bleeding and received multiple blood transfusions; she was given repeated courses of steroids, immunosuppression, immunoglobulin, and splenectomy without success

- Eventually, she was found to be profoundly hypothyroid and on correction of her thyroid deficiency the platelet count returned to normal and all bleeding stopped

years on thyroxine, the platelet count remains within the normal range. This suggests that the hypothyroidism may have resulted in the thrombocytopenia and as such our patient must have had subclinical thyroid deficiency for at least three years. No thyroid function tests were performed during this period to confirm this suggestion, although undiagnosed hypothyroidism is a common problem, and may affect up to $10 \%$ of the population. ${ }^{3}$ Macrocytosis might have suggested hypothyroidism; however, in the context of chronic blood loss and monthly blood transfusions the normal mean cell volume is not helpful. Hypothyroidism is known to be associated with acquired von Willebrand's disease. The activated partial thromboplastin time was consistently normal in our patient, although specific assays for von Willebrand factor were not performed at the time. von Willebrand factor deficiency may have contributed to the severe bleeding problems, although the bleeding that occurred would also be entirely consistent with the degree of thrombocytopenia alone. It is not clear why correction of thyroid hormone deficiency should result in normalisation of the platelet count. Autoantibodies to platelet antigens have been shown to coexist with autoimmune thyroid disease ${ }^{4}$ and, given our patient's normal bone marrow morphology and the documented failure to increment with platelet transfusion while thrombocytopenic, we suggest that the mechanism is probably improved platelet survival as a result of reduced platelet "autoimmunity", rather than increased platelet production.

\section{Authors' affiliations}

K M Bowles, G E Turner, J Z Wimperis, Department of Haematology, Norfolk and Norwich University NHS Trust, Colney Lane, Norwich, Norfolk NR4 7UY, UK

Correspondence to: Dr J Wimperis, Department of Haematology, Norfolk and Norwich University NHS Trust, Colney Lane, Norwich, Norfolk NR4 7UY, UK; jennie.wimperis@nnuh.nhs.uk

Accepted for publication 2 April 2004

\section{REFERENCES}

1 Guidelines for the investigation and management of idiopathic thrombocytopenic purpura in adults, children and in pregnancy. British Committee for Standards in Haematology General Haematology Task Force. Br J Haematol 2003; 120:574-96.

2 George JN, Woolf SH, Raskob GE, et al. Idiopathic thrombocytopenic purpura: a practice guideline developed by explicit methods for The American Society of Hematology (published on line at http://hematology.org/practice/ idiopathic.cfm)

3 Canaris GJ, Manowitz NR, Mayor G, et al. The Colorado thyroid disease prevalence study. Arch Intern Med 2000;160:526-34.

4 Cordiano I, Betterle C, Spadaccino C, et al. Autoimmune thrombocytopenia (AITP) and thyroid autoimmune disease (TAD): overlapping syndromes? Clin Exp Immunol 1998;113:373-8. 